\title{
Asymptotic Solutions of the Wave Equation with Degenerate Velocity and with Right-Hand Side Localized in Space and Time
}

\author{
Anatoly Anikin, Sergey Dobrokhotov, and \\ Vladimir Nazaikinskii \\ Dedicated to the 95th anniversary of V.A. Marchenko, \\ a prominent scientist and great personality
}

We study the Cauchy problem for the inhomogeneous two-dimensional wave equation with variable coefficients and zero initial data. The righthand side is assumed to be localized in space and time. The equation is considered in a domain with a boundary (shore). The velocity is assumed to vanish on the shore as a square root of the distance to the shore, that is, the wave equation has a singularity on the curve. This curve determines the boundary of the domain where the problem is studied. The main result of the paper is efficient asymptotic formulas for the solution of this problem, including the neighborhood of the shore.

Key words: wave equation, asymptotic solution, Maslov's canonical operator.

Mathematical Subject Classification 2010: 34E20, 35L05, 35Q35.

\section{Introduction}

In a finite domain $\Omega \subset \mathbb{R}^{2}$ with smooth boundary $\partial \Omega$, consider the Cauchy problem for the wave equation degenerating on $\partial \Omega$ :

$$
\begin{gathered}
\eta_{t t}-\left\langle\nabla, c^{2}(x) \nabla\right\rangle \eta=F(x, t),\left.\quad \eta\right|_{t=0}=0,\left.\quad \eta_{t}\right|_{t=0}=0, \\
F(x, t)=\lambda^{2} g^{\prime}(\lambda t)(\mathrm{e} V)\left(\frac{x-x_{0}}{\mu}\right), \quad t \in[0, T],
\end{gathered}
$$

where $c^{2}(x) \in C^{\infty}(\bar{\Omega})$ is a given function such that

$$
c^{2}(x)>0, x \in \Omega, \quad \text { and } \quad c^{2}(x)=0, \nabla c^{2}(x) \neq 0, x \in \partial \Omega,
$$

$\mu$ and $\lambda^{-1}$ are positive small parameters such that

$$
\lambda \mu>\text { const }>0
$$

(C) Anatoly Anikin, Sergey Dobrokhotov, and Vladimir Nazaikinskii, 2018 
$V(y)$ and $g(\tau)$ are smooth functions decaying as $|y| \rightarrow \infty$ and $\tau \rightarrow \infty, g(0)=0$, and $\mathrm{e}(z)$ is a smooth cutoff function; $\mathrm{e}(z)=1$ for $|z| \leq z_{0}$ and $\mathrm{e}(z)=0$ for $|z| \geq$ $z_{1}>z_{0}>0$. Actually, the choice of the cutoff function e is not important, and we introduce it only for mathematical rigor. We assume also that

$$
\int_{0}^{\infty} g(\tau) d \tau=1
$$

this gives $\lambda^{2} g^{\prime}(\lambda t) \rightarrow \delta(t-(+0))$ as $\lambda \rightarrow \infty$. Then it is easy to show that in the limit case $\lambda \rightarrow \infty$, the solution to (1.1), (1.2) becomes the solution to the homogenous equation (1.1)

$$
\begin{gathered}
\eta_{t t}-\left\langle\nabla, c^{2}(x) \nabla\right\rangle \eta=0, \quad x \in \bar{\Omega}, \quad t \in[0, T], \\
\left.\eta\right|_{t=0}=(\mathrm{e} V)\left(\frac{x-x_{0}}{\mu}\right),\left.\quad \eta_{t}\right|_{t=0}=0 .
\end{gathered}
$$

Later on, we will choose the functions $V$ and $g$ more specifically. Typical forms of the graphs of these functions are shown in Fig. 1.1.

This problem describes in the linear approximation the propagation of long waves (tsunami waves) created by a localized source and their run-up on the shore $[18,23,26,27]$. In this case, in dimensional variables one has $c^{2}(x)=\mathbf{g} D(x)$, where $D(x)$ is the basin depth, $\mathbf{g}$ is the free fall acceleration, and the right-hand side of the wave equation can be represented in the form $f\left(\frac{x-x_{0}}{l}, \frac{t}{t_{0}}\right)$, where $l$ is the characteristic size of the source and $t_{0}$ is the characteristic time of the source action. Let $L$ be the characteristic size of the basin, and let $D_{0}=D\left(x_{0}\right)$ be the characteristic depth in a neighborhood of the point $x_{0}$. We can pass from the dimensional variables $x, t, x_{0}$ to the dimensionless variables $x^{\prime}, t^{\prime}, x_{0}^{\prime}$ by setting $x=L x^{\prime}, t=T t^{\prime}, x_{0}=L x_{0}^{\prime}$, where $T=\frac{L}{\sqrt{\mathrm{g} D_{0}}}$. Then the dimensionless velocity takes the form $c^{\prime}=\sqrt{\frac{D}{D_{0}}}$, and the right-hand side becomes $f\left(\frac{x^{\prime}-x_{0}^{\prime}}{\mu}, \lambda t\right), \mu=$ $\frac{l}{L}, \lambda=\frac{T}{t_{0}}$. Consider the case where $L \approx 2000 \mathrm{~km}, l \approx 100 \mathrm{~km}, D_{0} \approx 4 \mathrm{~km}$, and $t_{0} \approx 10 \mathrm{~min}=600 \mathrm{sec}$; then $\mu \approx 1 / 20=0.05$ and $\lambda^{-1} \approx 0.06$ (because $\mathbf{g} \approx$ $\left.0.01 \mathrm{~km} / \mathrm{sec}^{2}\right)$. We see that the parameters $\mu$ and $\lambda^{-1}$ are close to each other, and hence assumption (1.4) holds in a typical physical situation. In tsunami wave theory, problem (1.5), (1.6) is known as the "piston model" (see [23]). Thus, the analysis of solutions of equation (1.1) permits one to understand how the source duration affects the shape of the generated wave.

According to [22], to fix a unique solution of (1.1), (1.2), one cannot set standard boundary conditions on $\partial \Omega$; instead, one needs to choose a self-adjoint extension of the corresponding operator. In water wave theory, it is natural to use the Friedrichs extension; this means that the energy integral

$$
J^{2}(t)=\frac{1}{2}\left(\left(\nabla \eta, c^{2}(x) \nabla \eta\right)_{L^{2}(\Omega)}+\left\|\eta_{t}\right\|_{L^{2}(\Omega)}^{2}\right)
$$

is finite.

Our goal is to construct an efficient asymptotic formula for the solution to problem (1.1), (1.2) as $\mu \rightarrow 0$ in a neighborhood of $\partial \Omega$. By "efficient" we mean 
that this formula can be used as a fast algorithm suitable for the computer implementation using software like Wolfram Mathematica or MatLab. Taking into account this goal, we choose the functions $V$ and $g$ as follows:

$$
\begin{gathered}
V(y)=W_{0}\left(T_{\psi_{0}} y\right), \\
g(\tau)=e^{-\tau} P_{n}(\tau), \quad P_{n}=\sum_{j=1}^{n} \frac{a_{j} \tau^{j}}{j !}, \quad \sum_{j=1}^{n} a_{j}=1, \quad a_{j} \in \mathbb{R},
\end{gathered}
$$

where

$$
W_{0}(y)=\frac{A}{\left[1+\left(y_{1} / b_{1}\right)^{2}+\left(y_{2} / b_{2}\right)^{2}\right]^{3 / 2}}, \quad b_{1} \geq b_{2}>0, T_{\psi_{0}}=\left(\begin{array}{cc}
\cos \psi_{0} & \sin \psi_{0} \\
-\sin \psi_{0} & \cos \psi_{0}
\end{array}\right) .
$$

The simplest two cases are $g(\tau)=\tau e^{-\tau}$ and $g(\tau)=\left((1-a) \tau+\frac{a}{2} \tau^{2}\right) e^{-\tau}$, where $a$ is a real parameter.
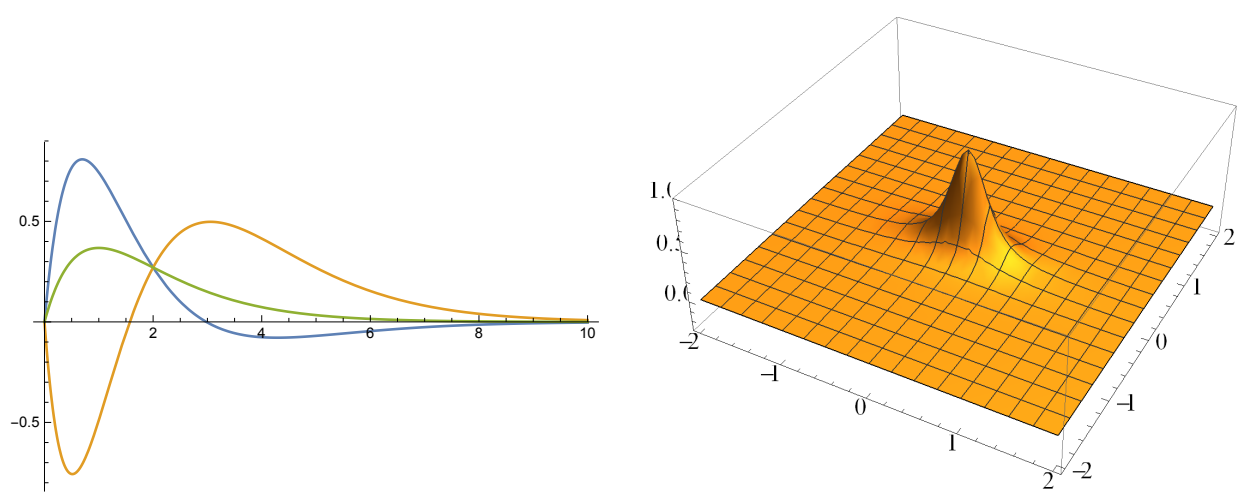

Fig. 1.1: Left: possible functions $g(\tau)$; right: a possible function $V$.

The source (1.7), which we call the simple source, was used in $[13,25,30]$, and its use leads to a significant simplification of formulas. The reason is that its Fourier transform is very simple,

$$
\tilde{V}(\rho \mathbf{n}(\psi))=A b_{1} b_{2} e^{-\rho \beta(\psi)}, \quad \beta(\psi)=\sqrt{b_{1}^{2} \cos ^{2}\left(\psi-\psi_{0}\right)+b_{2}^{2} \sin ^{2}\left(\psi-\psi_{0}\right)} .
$$

Needless to say, the real problem on the run-up of long waves on the shore is nonlinear and extremely complicated. The linear problem under consideration here is only a step towards its complete investigation. Until recently, analytical studies were carried out only in one-dimensional situation and for linear dependence of the bottom of the variable $x$. Here we should mention the fundamental work $[2,24]$, where some formulas were obtained for important solutions and the relationship between the solutions of linear and nonlinear problems (in the shallow water approximation) was actually established (see also [5, 6, 28]). Two-dimensional problems, of course, are more complicated: even in the linear situation it is clear that they have a nonstandard formulation: they lack the usual 
boundary conditions, which, in particular, leads to big difficulties in their numerical analysis. (One can find the latest achievements got in the field of numerical analysis in [17].) To the authors' knowledge, analytical studies in this area after paper [22] have appeared only recently in the works of the authors of this paper $[1,7-10]$. At the same time, it turned out that objects and constructions that seemed to be very far from the considered problem appear in problems with degenerate velocity.

The asymptotics as $\mu \rightarrow 0$ for the solution of (1.5), (1.6) was studied in several papers $[1,7-10,29]$ using a far-reaching generalization of the Maslov's canonical operator [14]. Owing to the degeneracy of $c(x)$ on $\partial \Omega$ (see (1.3)), the boundary becomes a caustic, and the characteristics intersecting it are nonstandard. Trajectories of the Hamiltonian system with the Hamiltonian function $H=c(x)|p|$ should be studied not in the "standard" phase space $\boldsymbol{\Phi}=T^{*} \Omega$, but in the extended one, $\boldsymbol{\Phi}=T^{*} \Omega \cup \boldsymbol{\Phi}_{\infty}$, where, informally speaking, $\boldsymbol{\Phi}_{\infty}$ is a hypersurface consisting of points with infinite momentum over $\partial \Omega$. The idea of the extended phase space was suggested in [20].

Further, one needs to use the modified Maslov's canonical operator [21] on the invariant Lagrangian manifold considered in the extended phase space. The modified canonical operator is a generalization of the standard canonical operator [14] based on Fock's procedure of quantized canonical transformations [15].

To write out the asymptotics, one should represent (following $[12,19]$ ) the initial data as some parametric integral of the standard canonical operator. Then the asymptotics of the solution is expressed as a similar parametric integral of the modified canonical operator. This general formula can be found in [7]. Inside the domain $\Omega$, this formula can be expressed in terms of the standard Maslov's canonical operator, which leads to a formula obtained earlier in [11]. The form of the solution on the boundary was studied in [7], and then in a neighborhood of the boundary in [1]. Under the additional assumption that the source is simple (1.7) and outside strong focal points on $\partial \Omega$ (which we define below) the asymptotic formula becomes very simple (free of any integrations).

The solution of the inhomogeneous problem was studied in detail in [4] (but outside the shore). It was shown there that problem (1.1), (1.2) can be reduced to the homogeneous problem (1.5), (1.6) with some special source called the equivalent source. In the present paper, we combine the results from [1] and [4] to obtain the asymptotic solution of problem (1.1), (1.2) in a neighborhood of $\partial \Omega$ outside strong focal points. The formula contains integration of Bessel functions and Fourier transforms of the functions $V$ and $g$. Using the argument from [3], we approximate this integral by a finite formula, which provides a good working algorithm for computer implementation valid in a neighborhood of $\partial \Omega$. We also provide numerics showing that this approximation is plausible.

The main result of this paper is the asymptotic formulas for the solutions of the inhomogeneous problem (1.1), (1.2); they are presented in Subsections 4.24.4: in Subsection 4.2, we write out a new general formula for the solution of the inhomogeneous problem, in Subsection 4.3 we discuss the approximation argument, and in Subsection 4.4 we show numerically that the approximation is valid. 
For completeness and better understanding our new results, we briefly recall some definitions and geometric constructions from [1,6-10,20,21] in Section 2 and the asymptotic formula for the homogeneous problem from the above-mentioned papers, in Section 3. In Subsection 4.1, we recall the construction of the equivalent source from $[3,4,6]$.

Finally, we point out that due to a good choice of the functions, defining the source generating waves, a significant part of complicated mathematical structures disappears in the obtained asymptotics, and the resulting formulas can be used by specialists without special mathematical education.

\section{Necessary constructions}

Let $x=\left(x_{1}, x_{2}\right)$ be Cartesian coordinates in $\Omega$, and $p=\left(p_{1}, p_{2}\right)$ be canonically conjugate momenta. The extended phase space $\boldsymbol{\Phi}$ introduced in [21] is defined as follows. Let $x_{*} \in \partial \Omega$ be arbitrary. Denote by $\pi_{\boldsymbol{\Phi}}: \mathbf{\Phi} \rightarrow \bar{\Omega}$ the projection onto the closure of $\Omega$. Let us describe $\boldsymbol{\Phi}$ and $\pi_{\boldsymbol{\Phi}}$. Without loss of generality, we may assume that the domain $\Omega$ near $x_{*}$ is determined by the inequality $x_{1}>f\left(x_{2}\right)$ for some smooth function $f\left(x_{2}\right)$. Then in a neighborhood of the set $\pi_{\boldsymbol{\Phi}}^{-1}\left(x_{*}\right)$ one can introduce local coordinates $(q, y, \theta, \xi)$ on $\boldsymbol{\Phi}$ such that the set $\boldsymbol{\Phi}_{\infty} \equiv \pi_{\boldsymbol{\Phi}}^{-1} \partial \Omega$ is given by the equality $q=0$, and for $q \neq 0$ (i.e., on $\boldsymbol{\Phi} \backslash \boldsymbol{\Phi}_{\infty}$ ) the introduced coordinates are expressed via $(x, p)$ by the formulas:

$$
x_{1}=f(y)+q^{2} \theta, \quad x_{2}=y, \quad p_{1}=q^{-1}, \quad p_{2}=\xi-q^{-1} f^{\prime}(y) .
$$

The manifold $\boldsymbol{\Phi}$ is symplectic with the closed (and, in fact, exact) 2 -form $\omega^{2}=$ $d \omega^{1}$ :

$$
\omega^{2}=d p \wedge d x=d \theta \wedge d q+d \xi \wedge d y, \quad \omega^{1}=p d x=2 \theta d q+q d \theta+\xi d y .
$$

The Lagrangian manifold $\Lambda_{2}$ associated with problem (1.5), (1.6) is constructed as follows. Let $\Lambda_{1}$ be a smooth curve given by the equalities: $x=$ $x_{0},|p|=1$. The set $\Lambda_{2}$ consists of all trajectories of the Hamiltonian system with the Hamiltonian function $H=c(x)|p|$ issued from $\Lambda_{1}$. Since the Hamiltonian vector field is nowhere tangent to $\Lambda_{1}$, the set $\Lambda_{2}$ forms (at least locally) the 2dimensional smooth manifold. Let us introduce the coordinates $\alpha=(\tau, \psi)$ on $\Lambda_{2}$. Here $\psi$ singles out a trajectory by the initial value of the momentum $\left.p\right|_{t=0}=$ $\mathbf{n}(\psi)=(\cos \psi, \sin \psi)$, and $\tau=c_{0} t$ is a parameter along the trajectory (where $c_{0}=$ $\left.c\left(x_{0}\right)\right)$. Actually, $(\tau, \psi)$ are the eikonal coordinates, and $\tau$ is the action (eikonal).

The manifold $\Lambda_{2}$ is given in the coordinates $x, p$ or $q, y, \theta, \xi$ by the equalities:

$$
\begin{aligned}
& x=X(\alpha), p=P(\alpha), \\
& q=Q(\alpha), y=Y(\alpha), \theta=\Theta(\alpha), \xi=\Xi(\alpha) .
\end{aligned}
$$

The representation (2.1) works everywhere on $\Lambda_{2}$ except for the set $\Lambda_{\infty}=\Lambda_{2} \cap$ $\boldsymbol{\Phi}_{\infty}$, while (2.2) works in a neighborhood of $\Lambda_{\infty}$.

A point $\alpha \in \Lambda_{2}$ is called focal, if

$$
J(\alpha):=\operatorname{det} \frac{\partial X}{\partial \alpha}(\alpha)=0
$$


(actually this definition does not depend on the specific choice of local coordinates $\alpha$ on $\left.\Lambda_{2}\right)$.

Any point $\alpha \in \Lambda_{\infty}$ is focal, since in a neighborhood of $\Lambda_{\infty}$, one has

$$
J(\alpha)=2 Q(\alpha) \Theta(\alpha) \operatorname{det} \frac{\partial(Q, Y)}{\partial \alpha}(\alpha)+Q^{2}(\alpha) J_{\xi y}(\alpha) \operatorname{det} \frac{\partial(\Theta, Y)}{\partial \alpha}(\alpha) .
$$

It follows from this equality that $J(\alpha) / Q(\alpha)$ can be continued onto $\Lambda_{\infty}$ as a smooth function. A focal point $\alpha \in \Lambda_{\infty}$ is called regular if $J(\alpha) / Q(\alpha) \neq 0$ and strong if $J(\alpha) / Q(\alpha)=0$.

It can be shown that in a neighborhood of each point $\alpha \in \Lambda_{\infty}$ one can choose either $(q, y)$ or $(q, \xi)$ as local coordinates. In other words at each point $\alpha$ one of the Jacobians: $J_{q y}=\operatorname{det} \frac{\partial(Q, Y)}{\partial \alpha}(\alpha)$ and $J_{q \xi}=\operatorname{det} \frac{\partial(Q, \Xi)}{\partial \alpha}(\alpha)$ does not vanish. Points where $J_{q y}(\alpha)$ vanishes are exactly strong focal points. Thus in a neighborhood of regular focal points one can always choose local coordinates $(q, y)$.

\section{Localized solution for homogeneous equation}

In this section we write out the asymptotics for the solution of the Cauchy problem (1.5), (1.6) in a neighborhood of a regular focal point on $\partial \Omega$.

Now let $\alpha_{*} \in \Lambda_{\infty}$ be a regular focal point, and $U \ni \alpha_{*}$ be a sufficiently small canonical chart on $\Lambda$ with coordinates $(q, y)$. Then the functions

$$
\Sigma(\alpha)=2 Q(\alpha) \sqrt{\Theta(\alpha)}
$$

and $Y(\alpha)$ define local coordinates in $U$, since the Jacobian $J_{\sigma y}(\alpha)=\operatorname{det} \frac{\partial(\Sigma, Y)}{\partial \mu}(\alpha)$ does not vanish at $\alpha_{*}$. Now recall that $\alpha=(\tau, \psi)$, and let us express $\tau=$ $\tau(\sigma, y), \psi=\psi(\sigma, y)$ from the equalities $\sigma=\Sigma(\tau, \psi), y=Y(\tau, \psi)$. Define also

$$
\tau_{\text {odd }}(\sigma, y)=\frac{\tau(\sigma, y)-\tau(-\sigma, y)}{2}, \quad \tau_{\text {ev }}(\sigma, y)=\frac{\tau(\sigma, y)+\tau(-\sigma, y)}{2} .
$$

Let $m$ be the index of the canonical chart $(U,(q, y))$ defined in $[1,7,10,21]$. Let $\mathrm{e}(\tau)$ be a compactly supported function equal to 1 in a neighborhood of zero, and let

$$
\widetilde{V}(k)=\frac{1}{2 \pi} \int_{\mathbb{R}^{2}} e^{-i\langle k, z\rangle} V(z) d z
$$

be the Fourier transform of the function $V(z)$. Finally, the following linear combination

$$
\mathbf{J}(v)=\mathbf{J}_{0}(v)+i \mathbf{J}_{1}(v)
$$

of Bessel's functions of the first kind $\mathbf{J}_{0}(v)$ and $\mathbf{J}_{1}(v)$ will play an important role below. Note that they naturally appear in theory of wave run-up on the shore $[2,16,28]$.

Theorem $3.1([1])$. The leading term $\eta_{\mathrm{hom}}^{U}(x, t)$ as $\mu \rightarrow 0$ of the asymptotic solution of problem $(1.5),(1.6)$ in a canonical chart $(U,(q, y))$ reads as follows:

$$
\eta_{\text {hom }}^{U}(x, t)=\widetilde{\eta}_{\text {hom }}\left(2 \sqrt{x_{1}-f\left(x_{2}\right)}, x_{2}, t\right)+\widetilde{\eta}_{\text {hom }}\left(-2 \sqrt{x_{1}-f\left(x_{2}\right)}, x_{2}, t\right),
$$


where

$$
\begin{aligned}
& \widetilde{\eta}_{\text {hom }}(\sigma, y, t)=\left\{\left(\frac{\tau_{\text {odd }}}{2 \sigma\left|J_{\sigma y}(\tau, \psi)\right|}\right)^{1 / 2} \mathrm{e}\left(\tau-c_{0} t\right)\right.
\end{aligned}
$$

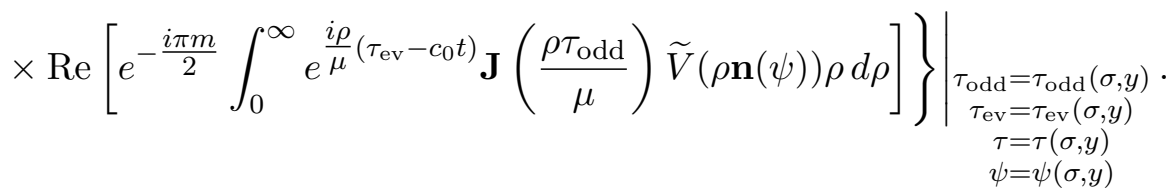

Notice that the function $\tau_{\text {odd }}(\sigma, y) / \sigma$ has no singularity on $\partial \Omega$ (where $\sigma=$ $0)$. In fact, this function is smooth and strictly positive in $U$. The same is true about the square root of it.

Now we additionally assume that the source is simple.

Theorem 3.2. If $V$ has the form (1.7), then the leading term of the asymptotic solution of problem (1.5), (1.6) in a canonical chart $U$ reads as follows:

$$
\begin{aligned}
& \widetilde{\eta}_{\text {sim }}^{U}(x, t)=\widetilde{\eta}_{\text {sim }}\left(2 \sqrt{x_{1}-f\left(x_{2}\right)}, x_{2}, t\right)+\widetilde{\eta}_{\text {sim }}\left(-2 \sqrt{x_{1}-f\left(x_{2}\right)}, x_{2}, t\right) \\
& \widetilde{\eta}_{\text {sim }}(\sigma, y, t)=A b_{1} b_{2}\left(\frac{\tau_{\text {odd }}(\sigma, y)}{2 \sigma\left|J_{\sigma y}(\tau(\sigma, y), \psi(\sigma, y))\right|}\right)^{1 / 2} \mathrm{e}\left(\tau(\sigma, y)-c_{0} t\right) \\
& \times \operatorname{Re}\left[e^{-\frac{i \pi m}{2}} \frac{\beta(\psi(\sigma, y))+\frac{i}{\mu}\left(-\tau(-\sigma, y)+c_{0} t\right)}{\left(\left(-\beta(\psi(\sigma, y))+\frac{i}{\mu}\left(\tau_{\text {ev }}(\sigma, y)-c_{0} t\right)\right)^{2}+\frac{\left(\tau_{\text {odd }}(\sigma, y)\right)^{2}}{\mu^{2}}\right)^{3 / 2}}\right] .
\end{aligned}
$$

In (3.4), let us set $\sigma=0$ and get the formula on the boundary (obtained earlier in [7]). We will use the fact that $\frac{\partial \tau(\sigma, y)}{\partial \sigma}=\sqrt{\Theta(\tau(\sigma, y), \psi(\sigma, y))}$ (see [1]). For simplicity, $\Theta(\tau(0, y), \psi(0, y))$ will be replaced by $\Theta(y)$. Then

$$
\begin{aligned}
\widetilde{\eta}_{\text {sim }}^{U}\left(0, x_{2}, t\right) & =\widetilde{\eta}_{\text {sim }}(0, y, t) \\
& =\left.\frac{A b_{1} b_{2} \Theta^{1 / 4}(y)}{\left|J_{\sigma y}(\tau, \psi)\right|^{1 / 2}} \mathrm{e}\left(\tau-c_{0} t\right) \operatorname{Re}\left(\frac{e^{-\frac{i \pi m}{2}}}{\gamma^{2}(\tau, \psi, t)}\right)\right|_{\substack{\tau=\tau(0, y) \\
\psi=\psi(0, y)}},
\end{aligned}
$$

where $\gamma(\tau, \psi, t)=-\beta(\psi)+\frac{i\left(\tau-c_{0} t\right)}{\mu}$.

\section{Localized solution for inhomogeneous equation}

4.1. Equivalent source. Consider inhomogeneous problem (1.1), (1.2). Let us set $\Lambda=\frac{\lambda \mu}{c_{0}}$. It is shown in [4] that the solution $\eta(x, t)$ can be split into the propagating and the transient parts: $\eta=\eta_{\text {prop }}+\eta_{\text {trans }}$. The transient part $\eta_{\text {trans }}$ is localized near $x=x_{0}$, and it decays exponentially with time (for details, see Theorem 3.1 from [4]). So the asymptotic behavior of the solution sufficiently far from $x_{0}$ is close to that of the propagating part. 
As to $\eta_{\text {prop }}$, it turns out to be close to the solution of the Cauchy problem for (1.2) with the special initial data called "the equivalent source". Consider the function $\widetilde{\eta}_{\text {prop }}=\eta_{1}+\frac{\partial \eta_{2}}{\partial t}$, where

$$
\left.\eta_{j}\right|_{t=0}=U_{j}\left(\frac{x-x_{0}}{\mu}\right),\left.\quad \frac{\partial \eta_{j}}{\partial t}\right|_{t=0}=0, \quad j=1,2,
$$

and the Fourier transforms of $U_{j}\left(\left(x-x_{0}\right) / \mu\right)$ are

$$
\widetilde{U}_{1}(p)=\sqrt{2 \pi} \operatorname{Re}\left[\widetilde{g}\left(\frac{|p|}{\Lambda}\right)\right] \widetilde{V}(p), \quad \widetilde{U}_{2}(p)=\frac{\sqrt{2 \pi}}{\lambda|p|} \operatorname{Im}\left[\widetilde{g}\left(\frac{|p|}{\Lambda}\right)\right] \widetilde{V}(p) .
$$

It turns out that $\eta_{\text {prop }}-\widetilde{\eta}_{\text {prop }}$ is $O(\mu)$ in the energy norm (see Theorem 3.4 and Remark 3.6 in [4]).

Strictly speaking, the results from [4] work for the domain $\Omega=\mathbb{R}^{2}$. However, they still can be used for computing the asymptotics near the shore if the source is located at a finite distance from it.

In what follows, by $\eta$ we will always mean $\widetilde{\eta}_{\text {prop }}$.

4.2. General formula for solution. After reducing the inhomogeneous problem to the homogeneous one, we can apply Theorem 3.1 to the problem (1.5), (4.1), (4.2) which immediately yields

Theorem 4.1. The leading term $\eta_{\text {inh }}^{U}(x, t)$ of the asymptotic solution of problem (1.1), (1.2) in a canonical chart $(U,(q, y))$ reads as follows:

$$
\eta_{\text {inh }}^{U}(x, t)=\widetilde{\eta}_{\text {inh }}\left(2 \sqrt{x_{1}-f\left(x_{2}\right)}, x_{2}, t\right)+\widetilde{\eta}_{\text {inh }}\left(-2 \sqrt{x_{1}-f\left(x_{2}\right)}, x_{2}, t\right),
$$

where

$$
\begin{aligned}
& \widetilde{\eta}_{\text {inh }}(\sigma, y, t)=\left\{( \frac { \pi \tau _ { \text { odd } } } { \sigma | J _ { \sigma y } ( \tau , \psi ) | } ) ^ { 1 / 2 } \mathrm { e } ( \tau - c _ { 0 } t ) \operatorname { R e } \left[e^{-\frac{i \pi m}{2}}\right.\right. \\
& \left.\left.\times \int_{0}^{\infty} e^{\frac{i \rho}{\mu}\left(\tau_{\mathrm{ev}}-c_{0} t\right)} \mathbf{J}\left(\frac{\rho \tau_{\mathrm{odd}}}{\mu}\right) \overline{\widetilde{g}\left(\frac{\rho}{\Lambda}\right)} \widetilde{V}(\rho \mathbf{n}(\psi)) \rho d \rho\right]\right\}\left.\right|_{\begin{array}{l}
\tau_{\mathrm{odd}}=\tau_{\mathrm{odd}}(\sigma, y) \\
\tau_{\mathrm{ev}}=\tau_{\mathrm{ev}}(\sigma, y) \\
\tau=\tau(\sigma, y) \\
\psi=\psi(\sigma, y)
\end{array}}
\end{aligned}
$$

Here the bar stands for the complex conjugation.

The Fourier transform of $g(\tau)$ given by (1.8) is

$$
\widetilde{g}(\xi)=P\left(i \frac{\partial}{\partial \xi}\right) \frac{1}{1+i \xi}=\sum_{j=1}^{n} \frac{a_{j}}{(1+i \xi)^{j+1}} .
$$

Inserting this into (4.3), we arrive at a rather complicated integral. However, it can be simplified if we study the solution only on the boundary $\sigma=0$.

Let us restrict ourselves with the case $n=2$. Then 


$$
\begin{aligned}
\widetilde{\eta}_{\text {inh }}(0, y, t)=\left\{\frac { A b _ { 1 } b _ { 2 } \Theta ^ { 1 / 4 } ( y ) } { | J _ { \sigma y } ( \tau , \psi ) | ^ { 1 / 2 } } \mathrm { e } ( \tau - c _ { 0 } t ) \operatorname { R e } \left[e^{-\frac{i \pi m}{2}} \int_{0}^{\infty} e^{\rho \gamma(\tau, \psi, t)}\right.\right. \\
\left.\left.\times\left(\frac{a_{1}}{(1-i \rho / \Lambda)^{2}}+\frac{1-a_{1}}{(1-i \rho / \Lambda)^{3}}\right) \rho d \rho\right]\right\}\left.\right|_{\substack{\tau=\tau(0, y) \\
\psi=\psi(0, y)}} .
\end{aligned}
$$

By using integration by parts, this integral can be reduced to the special function $\operatorname{Ei}(z)$. Namely, we obtain

$$
\begin{aligned}
& \widetilde{\eta}_{\text {inh }}(0, y, t)=\frac{A b_{1} b_{2} \Theta^{1 / 4}(y)}{\left|J_{\sigma y}(\tau, \psi)\right|^{1 / 2}} \mathrm{e}\left(\tau-c_{0} t\right) \operatorname{Re}\left[e ^ { - \frac { i \pi m } { 2 } } \left(\frac{3 a_{1}-1}{2} \Lambda^{2}-\frac{\left(a_{1}-1\right) \Lambda^{3} \gamma i}{2}\right.\right. \\
& \left.\left.+\left(a_{1} \Lambda^{2}+i\left(1-2 a_{1}\right) \Lambda^{3} \gamma-\frac{\left(a_{1}-1\right) \Lambda^{4} \gamma^{2}}{2}\right) e^{-i \gamma \Lambda} \operatorname{Ei}(i \gamma \Lambda)\right)\right]\left.\right|_{\substack{\tau_{\mathrm{ev}}=\tau_{\mathrm{ev}}(0, y) \\
\tau=\tau(0, y) \\
\psi=\psi(0, y)}} . \quad(4.4)
\end{aligned}
$$

4.3. Polynomial approximation. Thus, we have obtained an explicit formula (4.4) for the solution on the boundary. Unfortunately, it is not very good for numerical computations. It contains unbounded terms as $\mu \rightarrow 0$, but the singularities cancel out each other.

To get a more useful formula, we can use a trick suggested in [3]. Let us approximate $\overline{\widetilde{g}(\rho / \Lambda)}$ by some polynomial $G_{N}(\rho)$ of degree $N$ such that $\rho \overline{\widetilde{g}(\rho / \Lambda)} \widetilde{V}(\rho \mathbf{n}(\psi))$ and $\rho \widetilde{G}_{N}(\rho) \widetilde{V}(\rho \mathbf{n}(\psi))$ are close to each other for all $\psi$ and $\rho \in[0, \infty)$. Taking into account $(1.9)$, it is natural to take $\widetilde{G}_{N}$ as a linear combination of Laguerre polynomials of degree $\leq N$. Let us denote by $\widetilde{\eta}_{\text {inh, } N(\sigma, y, t)}$ the right-hand side of (4.3) where $\overline{\widetilde{g}(\rho / \Lambda)}$ is replaced with $\widetilde{G}_{N}(\rho)$.

Since the inverse Fourier transform of $\widetilde{G}_{N}$ is a linear combination of derivatives of $\delta(\tau), \widetilde{\eta}_{\text {inh }, N}$ can be expressed as follows. Assume that $\widetilde{G}_{N}(\rho)=\sum_{m=0}^{N} s_{m} \rho^{m}$. Then

$$
\widetilde{\eta}_{\text {inh }, N}=\sum_{m=0}^{N} s_{m}\left(\frac{i \Lambda}{\lambda}\right)^{m} \frac{\partial^{m} \widetilde{\eta}_{\text {hom }}}{\partial t^{m}}=\sum_{m=0}^{N} s_{m}\left(\frac{i \mu}{c_{0}}\right)^{m} \frac{\partial^{m} \widetilde{\eta}_{\text {hom }}}{\partial t^{m}} .
$$

Inserting (3.4) here, we get the final approximate formula for the solution valid in a neighborhood of the boundary.

This formula can be further simplified on the boundary. Let us exclude the factor $\mathrm{e}\left(\tau-c_{0} t\right)$ from (3.5) and consider the solution only near the wave front (i.e., when $\tau-c_{0} t$ is sufficiently close to zero):

$$
\begin{aligned}
\widetilde{\eta}_{\text {inh }, N}(0, y, t)= & \frac{A b_{1} b_{2} \Theta^{1 / 4}(y)}{\left|J_{\sigma y}(\tau, \psi)\right|^{1 / 2}} \\
& \times\left.\operatorname{Re}\left[e^{-\frac{i \pi m}{2}} \sum_{m=0}^{N} \frac{(-1)^{m}(m+1) ! s_{m}}{\gamma^{m+2}(\tau, \psi, t)}\right]\right|_{\substack{\tau=\tau(0, y) \\
\psi=\psi(0, y)}}
\end{aligned}
$$


4.4. Numerical comparison. Our numerical computations show that the asymptotic solution (4.6) approximates the "exact" asymptotic solution (4.4) quite well. We have set the following values of the parameters:

$$
N=10, \quad \mu=0.1, \quad b_{1}=b_{2}=1, \quad \Lambda=1.5, \quad a_{1}=\frac{1}{2}, \quad m=0 .
$$

Define $\tilde{t}=c_{0} t-\tau$, then $\gamma \equiv \gamma(\tilde{t})=-\frac{i \tilde{t}}{\mu}-1$. Let $\eta_{1}(\tilde{t})$ and $\eta_{2}(\tilde{t})$ be the expressions in square brackets in (4.4) and (4.6), respectively. The functions $\rho \widetilde{\widetilde{g}(\rho / \Lambda)} \widetilde{V}(\rho \mathbf{n}(\psi))$ and $\rho \widetilde{G}_{N}(\rho) \widetilde{V}(\rho \mathbf{n}(\psi))$ are compared in Fig. 4.1, and the functions $\eta_{1}(\tilde{t})$ and $\eta_{2}(\tilde{t})$ are compared in Fig. 4.2. We see that the polynomial approximation is satisfactory on the boundary.

On the other hand, as numerical computations in [3] show, the polynomial approximation for the asymptotic solution is also satisfactory inside the domain $\Omega$. Combining these results, we may expect that formulas (4.5), (3.4) provide a good approximation for the solution in a neighborhood of the boundary.

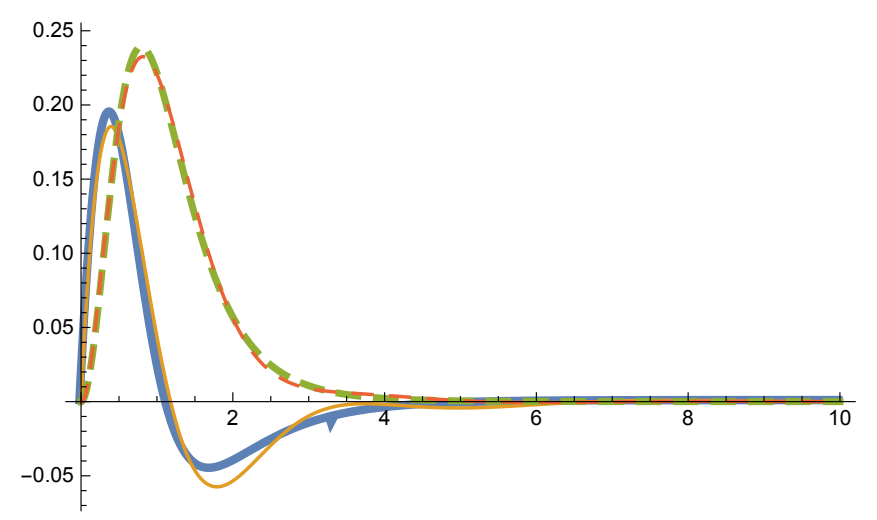

Fig. 4.1: Comparison of $\rho \overline{\widetilde{g}(\rho / \Lambda)} \widetilde{V}(\rho \mathbf{n}(\psi))$ (bold) and $\rho \widetilde{G}_{N}(\rho) \widetilde{V}(\rho \mathbf{n}(\psi))$ (plain). (Solid line for the real part, dashed line for the imaginary part.)

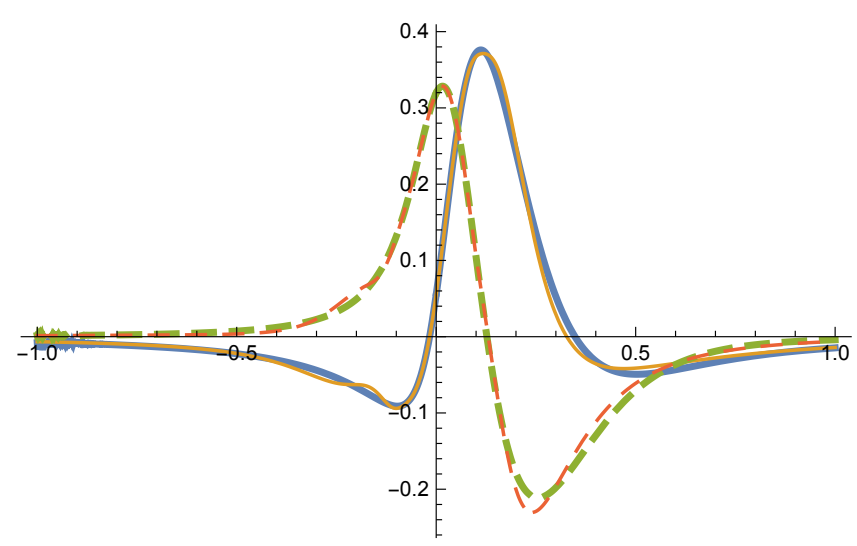

Fig. 4.2: Comparison of $\eta_{1}(\tilde{t})$ (bold) and $\eta_{2}(\tilde{t})$ (plain). (Solid line for the real part, dashed line for the imaginary part.) 
Supports. This work is supported by Russian Science Foundation, project No. 16-11-10282.

\section{References}

[1] A.Yu. Anikin, S.Yu. Dobrokhotov, and V.E. Nazaikinskii, Simple asymptotics for a generalized wave equation with degenerating velocity and their applications to the linearized long wave run-up problem, Mat. Zametki 104 (2018), No. 4, 483-504 (Russian); Engl. transl.: Math. Notes 104, No. 3-4 (2018), 471-488.

[2] G.F. Carrier and H.P. Greenspan, Water waves of finite amplitude on a sloping beach, J. Fluid Mech. 4 (1958), No. 1, 97-109.

[3] S.Yu. Dobrokhotov and A.Yu. Anikin, Approximation of solutions of the twodimensional wave equation with variable velocity and localized right-hand side using some "simple" solutions, Mat. Zametki 100 (2016), No. 6, 825-837 (Russian); Engl. transl.: Math. Notes 100 (2016), No. 6, 796-806.

[4] S.Yu. Dobrokhotov, D.S. Minenkov, V.E. Nazaikinskii, and B. Tirozzi, Functions of noncommuting operators in an asymptotic problem for a $2 D$ wave equation with variable velocity and localized right-hand side, Oper. Theory Adv. Appl., 228, Birkhauser, Basel, 2013, 95-126.

[5] S.Yu. Dobrokhotov, D.S. Minenkov, V.E. Nazaikinskii, and B. Tirozzi, Simple exact and asymptotic solutions of the $1 D$ run-up problem over a slowly varying (quasiplanar) bottom, Theory and Applications in Mathematical Physics, World Sci., Singapore, 2015, 29-47.

[6] S.Yu. Dobrokhotov and V.E. Nazaikinskii, Asymptotics of localized wave and vortex solutions of linearized shallow water equations, Actual Problems of Mechanics (the book of papers dedicated to the 50th anniversary of the Ishlinsky Institute for Problems in Mechanics RAS), Nauka, Moscow, 2015, 98-139 (Russian).

[7] S.Yu. Dobrokhotov and V.E. Nazaikinskii, Characteristics with singularities and the boundary values of the asymptotic solution of the Cauchy problem for a degenerate wave equation, Mat. Zametki 100 (2016), No. 5, 710-731 (Russian); Engl. transl.: Math. Notes 100 (2016), No. 5-6, 695-713.

[8] S.Yu. Dobrokhotov, V.E. Nazaikinskii, and B. Tirozzi, Asymptotic solution of the one-dimensional wave equation with localized initial data and with degenerating velocity. I, Russ. J. Math. Phys. 17 (2010), No. 4, 434-447.

[9] S.Yu. Dobrokhotov, V.E. Nazaikinskii, and B. Tirozzi, Asymptotic solutions of the two-dimensional model wave equation with degenerating velocity and localized initial data, Algebra Anal. 22 (2010), No. 6, 67-90 (Russian); Engl. transl.: St. Petersburg Math. J. 22 (2011), No. 6, 895-911.

[10] S.Yu. Dobrokhotov, V.E. Nazaikinskii, and B. Tirozzi, Two-dimensional wave equation with degeneration on the curvilinear boundary of the domain and asymptotic solutions with localized initial data, Russ. J. Math. Phys. 20 (2013), No. 4, 389-401.

[11] S. Dobrokhotov, A. Shafarevich, and B. Tirozzi, Localized wave and vortical solutions to linear hyperbolic systems and their application to linear shallow water equations, Russ. J. Math. Phys. 15 (2008), No. 2, 192-221.

[12] S.Yu. Dobrokhotov, B. Tirozzi, and A.I. Shafarevich, Representations of rapidly decreasing functions by the Maslov canonical operator, Mat. Zametki 82 (2007), No. 5, 792-796 (Russian); Engl. transl.: Math. Notes 82 (2007), No. 5-6, 713-717. 
[13] S.F. Dotsenko, B.Yu. Sergievskii and L.V. Cherkasov, Space tsunami waves generated by alternating displacement of the ocean surface, Tsunami Research 1 (1986), $7-14$.

[14] M.V. Fedoriuk and V.P. Maslov, Semi-Classical Approximation in Quantum Mechanics, Nauka, Moscow, 1976 (Russian); Engl. transl.: Reidel, Dordrecht, 1981.

[15] V. A. Fock, On the canonical transformation in classical and quantum mechanics, Vestn. Leningrad. Univ. 16 (1959), 67--70 (Russian); Engl. transl.: Acta Phys. Acad. Sci. Hungaricae 27 (1-4) (1969), 219-224.

[16] J.B. Keller and H.B. Keller, Water Wave Run-Up on a Beach, 1964, ONR, Research Report Contract No. NONR-3828(00), Dept. of the Navy, Washington, DC.

[17] A. Kozelkov, V. Efremov, A. Kurkin, E. Pelinovsky, N. Tarasova, and D. Strelets, Three-dimensional numerical simulation of tsunami waves based on Navier-Stokes equation, Science of Tsunami Hazards 36 (2017), No. 4, 45-58.

[18] C.C. Mei, The Applied Dynamics of Ocean Surface Waves, World Sci., Singapore, 1989

[19] V.E. Nazaikinskii, On the representations of localized functions in $R^{2}$ by the Maslov canonical operator, Mat. Zametki 96 (2014), No. 1, 88-100 (Russian); Enlg. transl.: Math. Notes 96 (2014), No. 1-2, 99-109.

[20] V.E. Nazaikinskii, Phase space geometry for a wave equation degenerating on the boundary of the domain, Mat. Zametki 92 (2012), No. 1, 153-156 (Russian); Engl. transl,: Math. Notes 92 (2012), No. 1-2, 144-148.

[21] V.E. Nazaikinskii, The Maslov canonical operator on Lagrangian manifolds in the phase space corresponding to a wave equation degenerating on the boundary, Mat. Zametki 96 (2014), No. 2, 261-276 (Russian); Engl. transl.: Math. Notes 96 (2014), No. 1-2, 248-260.

[22] O.A. Oleinik and E.V. Radkevic, Second order equations with nonnegative characteristic form, American mathematical society, Providence, Rhode Iland Plenum Press, New York-London, 1973.

[23] E.N. Pelinovskii, Hydrodynamics of Tsunami Waves, Inst. Prikl. Fiz., Nizhnii Novgorod, 1996 (Russian).

[24] E.N. Pelinovsky and R.Kh. Mazova, Exact analytical solutions of nonlinear problems of tsunami wave run-up on slopes with different profiles, Natural Hazards 6 (1992), No. 3, 227-249.

[25] S.Ya. Sekerzh-Zen'kovich, Simple asymptotic solution of the Cauchy-Poisson problem for head waves, Russ. J. Math. Phys. 16 (2009) No. 2, 315-322.

[26] Y.I. Shokin, L.B. Chubarov, An.G. Marchuk, and K.V. Simonov, Computational Experiment in the Tsunami Problem, Nauka, Novosibirsk, 1989 (Russian).

[27] J.J. Stoker, Water Waves: The Mathematical Theory with Applications, John Wiley and Sons, New York, 1992.

[28] C.E. Synolakis, On the roots of $f(z)=J_{0}(z)-i J_{1}(z)$, Quart. Appl. Math. 46, (1988), No. 1, 105-107.

[29] T. Vukašinac and P. Zhevandrov, Geometric asymptotics for a degenerate hyperbolic equation, Russ. J. Math. Phys. 9 (2002), No. 3, 371-381. 
[30] S. Wang, B. Le Mehaute, and Chia-Chi Lu, Effect of dispersion on impulsive waves, Marine Geophysical Researchers 9 (1987), 95-111.

Received March 19, 2018.

Anatoly Anikin,

Ishlinsky Institute for Problems in Mechanics RAS, pr. Vernadskogo, 101-1, Moscow, 119526, Russia;

Moscow Institute of Physics and Technology, Institutskiy per. 9, Dolgoprudny, Moscow Region, 141701, Russia,

E-mail: anikin83@inbox.ru

Sergey Dobrokhotov,

Ishlinsky Institute for Problems in Mechanics RAS, pr. Vernadskogo, 101-1, Moscow, 119526, Russia;

Moscow Institute of Physics and Technology, Institutskiy per. 9, Dolgoprudny, Moscow Region, 141701, Russia,

E-mail: nazaikinskii@yandex.ru

Vladimir Nazaikinskii,

Ishlinsky Institute for Problems in Mechanics RAS, pr. Vernadskogo, 101-1, Moscow, 119526, Russia;

Moscow Institute of Physics and Technology, Institutskiy per. 9, Dolgoprudny, Moscow Region, 141701, Russia,

E-mail: nazaikinskii@yandex.ru

\section{Асимптотичні розв'язки хвильового рівняння зі швидкістю, що вироджується, і з правою стороною, яка локалізована в просторі та часі}

Anatoly Anikin, Sergey Dobrokhotov, and Vladimir Nazaikinskii

Вивчається задача Коші для неоднорідного двовимірного хвильового рівняння зі змінними коефіцієнтами та нульовими початковими даними. Вважається, що права частина локалізована в просторі та часі. Рівняння розглядається в області з межею (берегом). Вважається, що швидкість на березі зникає як квадратний корінь відстані до берега, тобто хвильове рівняння має задану на кривій особливість. Ця крива і визначає межу області, в якій вивчається задача. Основний результат роботи - ефективні асимптотичні формули для розв'язку зазначеної задачі, включаючи окіл берега.

Ключові слова: хвильове рівняння, асимптотичний розв'язок, канонічний оператор Маслова. 